\title{
NONCOMMUTATIVE JORDAN DIVISION ALGEBRAS
}

\author{
BY \\ KEVIN MCCRIMMON( $\left.{ }^{1}\right)$
}

\begin{abstract}
The structure theory for noncommutative Jordan algebras with chain conditions leads to the following simple algebras: (I) division algebras, (II) forms of nodal algebras, (III) algebras of generic degree two, (IV) commutative Jordan matrix algebras, (V) quasi-associative algebras. The chain condition is always satisfied in a division algebra, hence does not serve as a finiteness restriction. Consequently, the general structure of noncommutative Jordan division algebras, even commutative Jordan division algebras, is unknown. In this paper we will classify those noncommutative Jordan division algebras which are forms of algebras of types (II)-(V); this includes in particular all the finite-dimensional ones.
\end{abstract}

We begin by recalling some basic results about noncommutative Jordan rings. We are interested only in simple rings, and simple rings can be considered as algebras over their centroids, which are fields, so we restrict ourselves to algebras over a field $\Phi$ (of arbitrary characteristic).

A noncommutative Jordan algebra $\mathfrak{A}$ over $\Phi$ is a linear algebra such that for any $x \in \mathfrak{A}$ the left and right multiplications by $x$ and $x^{2}$ commute: $L_{x}, R_{x}, L_{x^{2}}, R_{x^{2}}$ generate a commutative algebra of transformations on $\mathfrak{A}$ (and such that this continues to hold in any scalar extension $\mathfrak{A}_{\Omega}$ ). Any such algebra determines [8] a quadratic Jordan algebra $\mathfrak{A}^{+}$with $U$-operator

$$
U_{x}=L_{x}\left(L_{x}+R_{x}\right)-L_{x^{2}}=R_{x}\left(R_{x}+L_{x}\right)-R_{x^{2}} .
$$

An element $x \in \mathfrak{A}$ is invertible if there exists $y \in \mathfrak{A}$ with $x y=y x=1, x^{2} y=y x^{2}=x$; this is equivalent [5] to $x$ being invertible in $\mathfrak{A}^{+}$, or to $U_{x}$ being an invertible transformation. If all nonzero elements of $\mathfrak{A}$ are invertible we say $\mathfrak{A}$ is a noncommutative Jordan division algebra; this is equivalent to $\mathfrak{A}^{+}$being a quadratic Jordan division algebra.

Our definition differs from the more usual one in which all $L_{x}$ and $R_{x}$ are required to be invertible transformations. For finite-dimensional or, more generally, algebraic algebras our condition is less restrictive. Also, it has the desirable property that if $\mathfrak{A}$ is an associative division algebra then any mutation $\mathfrak{U}^{(\lambda)}$ with product $x \cdot y=\lambda x y+(1-\lambda) y x$ is a noncommutative Jordan division algebra. For example, if $\mathfrak{Q}$ is the division algebra of real quaternions then $\mathfrak{Q}^{+}=\mathfrak{Q}^{(1 / 2)}$ is a commutative Jordan division algebra in our sense, but not in the usual one because of the

Received by the editors February 25, 1971.

AMS 1969 subject classifications. Primary 1740.

Key words and phrases. Noncommutative Jordan algebra, division algebra.

( $\left.{ }^{1}\right)$ This research was partially supported by AFOSR Grant No. 335-63.

Copyright (C) 1972, American Mathematical Society 
presence of zero-divisors: $i \cdot j=\frac{1}{2}(i j+j i)=0$ for $i, j$ the usual quaternion basis elements.

According to the structure theory [5], [7], the simple noncommutative Jordan algebras with d.c.c. (on inner ideals) are those of the form

(I) Noncommutative Jordan division algebras.

(II) Certain nondivision algebras of degree one in which all elements are invertible or nilpotent.

(III) Degree two algebras $\mathfrak{A}(Q, S, \times)=\Phi e_{1} \oplus \Phi e_{2} \oplus \mathfrak{B}$ with multiplication

$$
\begin{gathered}
e_{i}^{2}=e_{i}, \quad e_{i} e_{j}=0, \quad e_{i} v=S_{i}(v), \quad v e_{i}=S_{j}(v), \\
v w=Q\left(S_{2} v, w\right) e_{1}+Q\left(S_{1} v, w\right) e_{2}+v \times w \quad(v, w \in \mathfrak{B})
\end{gathered}
$$

where $Q$ is a nondegenerate quadratic form on $\mathfrak{B}, S_{1}$ and $S_{2}$ are linear transformations on $\mathfrak{B}$, with $S_{1}+S_{2}=I$ and $Q\left(S_{i} v, v\right)=Q(v)$ for all $v \in \mathfrak{B}$, and $\times$ is an alternate bilinear product on $\mathfrak{B}$ with $v \times v=0, Q(v, v \times w)=0$ for all $v, w \in \mathfrak{B}$.

(IV) Commutative Jordan matrix algebras $\mathfrak{S}_{\mathfrak{c}}\left(\mathfrak{D}_{n}, \gamma\right)$ over a field of characteristic $\neq 2$, where $n \geqq 2$ and $\mathfrak{D}$ is either (i) a sum $\Delta \oplus \Delta^{\circ}$ of anti-isomorphic associative division algebras with exchange involution, (ii) an associative division algebra $\Delta$ with involution (where if $n=2$ we require $\mathfrak{S}\left(\Delta,{ }^{*}\right)$ generates $\Delta$ ), (iii) a split quaternion algebra $\Phi_{2}$ with standard involution (only for $n \geqq 3$ ), (iv) a Cayley algebra $\mathbb{E}$ with standard involution (only for $n=3$ ).

(V) Quasi-associative algebras $\mathfrak{S}\left(\mathfrak{D}_{n}, \gamma\right)^{(\lambda)}$ for $n \geqq 2, \lambda \neq \frac{1}{2}$ in the center of $\mathfrak{D}$ with $\lambda+\lambda^{*}=1$, and where $\mathfrak{D}$ is either (i) a sum $\Delta \oplus \Delta^{\circ}$ of anti-isomorphic associative division algebras with exchange involution, (ii) an associative division algebra $\Delta$ with involution of second kind.

Furthermore [9], in the finite-dimensional case the algebras of type (II) are forms of nodal algebras (algebras $\Omega 1+\mathfrak{N}$ where $\mathfrak{R}$ is a nil subspace but not an ideal):

(IIa) Forms of nodal algebras $\mathfrak{A}\left(F_{n}, C\right)$ of characteristic $p \neq 0,2$ where $\mathfrak{A}\left(F_{n}, C\right)$ is the truncated polynomial ring $F_{n}=\Omega\left[z_{1}, \ldots, z_{n}\right]\left(z_{i}^{p}=0\right)$ with multiplication

$$
f g=f \cdot g+f \times g, \quad f \times g=\sum c_{i j} \cdot \partial_{i} f \cdot \partial_{j} g,
$$

where $f \cdot g$ is the product in $F_{n}, c_{i j} \in F_{n}$ satisfy $c_{i j}+c_{j i}=0$, and $\partial_{i} f$ is the ordinary partial derivative $\partial f / \partial x_{i}$.

(IIb) Forms of commutative nodal algebra $\mathfrak{U}(\tau, \times)=\Omega 1+\mathfrak{N}$ of characteristic 2 with multiplication

$$
z w=\tau(z, w) 1+z \times w \quad(z, w \in \mathfrak{R})
$$

where $\tau$ is an alternate bilinear form and $\times$ an alternate product on $\mathfrak{R}, \tau(z, z)=0$ and $z \times z=0$.

1. Forms of commutative Jordan algebras. If $\mathfrak{A}$ is a form of a commutative Jordan matrix algebra $\mathfrak{A}_{\Omega}=\mathfrak{S}\left(\mathfrak{D}_{n}, \gamma\right)$ of type (IV) over a field $\Omega$ of characteristic $\neq 2, n \geqq 2$, then $\mathfrak{A}$ too must be commutative over a field $\Phi$ of characteristic $\neq 2$. The results in this case are well known. 
THEOREM 1. If the commutative Jordan division algebra $\mathfrak{A}$ of characteristic $\neq 2$ is a form of a Jordan matrix algebra of type (IV) then $\mathfrak{A}$ is isomorphic to one of

(i) $\Delta^{+}$,

(ii) the space $\mathfrak{S}\left(\Delta,{ }^{*}\right),{ }^{*}$ an involution on $\Delta$,

(iii) an exceptional division algebra $\Im(\Delta, \mu)$ or $\Im\left(\Delta, u, \mu,{ }^{*}\right)$ obtained from the First or Second Tits Construction [10, pp. 507-509]

where $\Delta$ is an associative division algebra.

Proof. Consider first the special case. Suppose $\mathfrak{A}$ is a commutative Jordan division algebra with $\mathfrak{A}_{\Omega}=\mathfrak{S}\left(\mathfrak{D}_{n}, \gamma\right)$ for $n \geqq 2$, $\mathfrak{D}$ associative (where $\mathfrak{S}\left(\mathfrak{D},{ }^{*}\right)$ generates $\mathscr{D}$ in case $n=2)$. By Martindale's Theorem [3, p. 141] $\mathfrak{A}_{\Omega}$ is special and reflexive: $\mathfrak{A}_{\Omega}=\mathfrak{S}\left(\operatorname{su}\left(\mathfrak{A}_{\Omega}\right), \pi\right)$ for su $\left(\mathfrak{A}_{\Omega}\right)$ the special universal envelope and $\pi$ the main involution. By universal nonsense [3, pp. 78-79] $\mathfrak{A}=\mathfrak{S}(\mathrm{su}(\mathfrak{A}), \pi)$ is also special and reflexive. Then [3, p. 166] either $\mathfrak{A}=\mathfrak{S}\left(\Delta,{ }^{*}\right)$ for $\Delta$ an associative division algebra with involution (case (ii) above) or $\mathfrak{A}=\mathfrak{H}\left(\Delta \oplus \Delta^{*},{ }^{*}\right) \cong \Delta^{+}$where $\Delta \oplus \Delta^{*}$ is a direct sum of two anti-isomorphic associative division algebras under the exchange involution (case (i)).

Now consider the exceptional case. If $\mathfrak{A}_{\Omega}=\mathfrak{S}\left(\mathfrak{C}_{3}\right)$ for $\mathfrak{C}$ a Cayley algebra then $\mathfrak{A}_{\Omega}$ (and hence $\mathfrak{A}$ ) is a 27-dimensional exceptional algebra. All such are obtained from the two Tits Constructions (see [1], [3, pp. 413-422], [11, p. 313]).

Conversely, if $\Delta$ is an associative division algebra such that $\Delta_{\Omega}=\mathscr{D}_{n}(n \geqq 2)$ for some extension $\Omega$, then $\mathfrak{A}=\Delta^{+}$or $\mathfrak{S}\left(\Delta,{ }^{*}\right)$ will be a Jordan division algebra which is a form of $\mathfrak{D}_{n}^{+}$or $\mathfrak{S}\left(\mathfrak{D}_{n},{ }^{*}\right)$ (in the latter case, when $n=2$ we must require $\mathfrak{S}\left(\mathfrak{D},{ }^{*}\right)$ generates $\mathfrak{D}$ in order to get an algebra of type (IV)). Similarly, if $\Delta_{\Omega}=\Omega_{3}$ for some extension (so $\Delta$ has generic degree 3 ) then $\Im(\Delta, \mu)$ or $\Im\left(\Delta, u, \mu,{ }^{*}\right)$ can be defined and is a form of $\mathfrak{S}\left(\mathfrak{S}_{3}\right)$; the condition that it be a Jordan division algebra is that $\mu$ is not a generic norm of an element of $\Delta$.

2. Forms of quasi-associative algebras. In this section we suppose $\mathfrak{A}$ is a noncommutative Jordan algebra which is quasi-associative, that is, a form of a split quasi-associative algebra $\mathfrak{D}^{(\lambda)}$ for $\mathscr{D}$ associative and $\lambda \neq \frac{1}{2}$. The results here will be completely general, with no finiteness conditions. Modifying the arguments of [6] to include characteristic 2 , we have

THEOREM 2. A noncommutative Jordan algebra over a field $\Phi$ is quasi-associative if and only if it is isomorphic to one of the following:

(i) $\mathfrak{D}^{(\lambda)}$,

(ii) $\mathfrak{S}\left(\mathfrak{D}^{(\lambda)}, *\right)$ for $*$ an involution of second kind on $\mathfrak{D}$ with $\lambda+\lambda^{*}=1$, where $\mathfrak{D}$ is an associative algebra over $\Phi$ and $\lambda \neq \frac{1}{2}$ an element in its center with $\lambda(1-\lambda) \in \Phi$.

Proof. Suppose $\mathfrak{A}$ is quasi-associative. If $\mathfrak{A}$ itself is associative, we have $\mathfrak{A}=\mathfrak{D}^{(\lambda)}$ for $\mathfrak{D}=\mathfrak{A}$ and $\lambda=1$. Otherwise, some associator $[x, y, z] \neq 0$. Regard $\mathfrak{A}$ as imbedded 
in $\mathfrak{A}_{\Sigma}=F^{(\lambda)}$ for $\mathbb{E}$ associative, $\lambda \neq \frac{1}{2}$ in $\Sigma$. Recall the formula

(2) $[x, y, z]^{(\lambda)}=\lambda(2 \lambda-1)[x, y, z]+(1-\lambda)(2 \lambda-1)[z, y, x]+\lambda(1-\lambda)[x, y, z]^{\circ}$

relating the associators

$$
\begin{aligned}
{[x, y, z] } & =(x y) z-x(y z), \\
{[x, y, z]^{(\lambda)} } & =\left(x \cdot{ }_{\lambda} y\right) \cdot{ }_{\lambda} z-x \cdot{ }_{\lambda}\left(y \cdot{ }_{\lambda} z\right),
\end{aligned}
$$

and

$$
[x, y, z]^{\circ}=(x \circ y) \circ z-x \circ(y \circ z)
$$

for $x \cdot{ }_{\lambda} y=\lambda x y+(1-\lambda) y x, x \circ y=x y+y x$. Applying this to $x, y, z \in \mathfrak{A} \subset \mathfrak{A}_{\Sigma}=\mathfrak{F}(\lambda)$, we have $[x, y, z]=[z, y, x]=0$ by the associativity of $\mathbb{E}$, so the fact that $[x, y, z]^{(\lambda)}$ and $[x, y, z]^{\circ}$ belong to $\mathfrak{A}$ if $x, y, z$ do implies $\lambda(1-\lambda) \in \Phi$. Let $\Omega=\Phi(\lambda)$ and $\mathfrak{D}=\left\{\mathfrak{A}_{\Omega}\right\}^{(\mu)}$ where $\mu=\lambda(2 \lambda-1)^{-1}$ satisfies $2 \lambda \mu=\lambda+\mu$. Then the general transitivity relation

$$
\left\{\mathfrak{B}^{(\mu)}\right\}^{(\lambda)}=\mathfrak{B}^{(\mu \odot \lambda)} \quad(\mu \odot \lambda=1-\mu-\lambda+2 \mu \lambda)
$$

for mutations shows $\mathfrak{A}_{\Omega}=\mathfrak{A}_{\Omega}^{(1)}=\mathfrak{A}_{\Omega}^{(\mu \odot \odot)}=\left\{\mathfrak{H}_{\Omega}^{(\mu)}\right\}^{(\lambda)}=\mathfrak{D}^{(\lambda)}$, where $\mathfrak{D}=\mathfrak{A}_{\Omega}^{(\mu)}$ is associative because $\mathfrak{D} \subset \mathfrak{U}_{\Sigma}^{(\mu)}=\mathfrak{F}^{(\lambda)(\mu)}=\mathscr{E}^{(\lambda \odot \mu)}=\mathscr{E}$ is associative.

If $\Omega=\Phi$ then $\mathfrak{A}=\mathfrak{D}^{(\lambda)}$ as in case (i). Suppose $\Omega=\Phi+\Phi \lambda=\Phi \lambda+\Phi(1-\lambda)$ for $\lambda(1-\lambda) \in \Phi$ where $\lambda \notin \Phi$; then $\Omega$ carries a nontrivial automorphism * of period two given by $\lambda^{*}=1-\lambda, \lambda+\lambda^{*}=1$ and this extends to an automorphism $* \otimes 1$ of period two on $\mathfrak{D}^{(\lambda)}=\mathfrak{A}_{\Omega}=\Omega \otimes \mathfrak{A}:(\omega \otimes a)^{*}=\omega^{*} \otimes a$. Since $\mathfrak{S}\left(\Omega,{ }^{*}\right)=\Phi$ we have $\mathfrak{S}\left(\mathfrak{A}_{\Omega},{ }^{*}\right)$ $=\Phi \otimes \mathfrak{U}=\mathfrak{A}$, so the subalgebra of $\mathfrak{A}_{\Omega}=\mathfrak{D}^{(\lambda)}$ fixed by * is just $\mathfrak{A}=\mathfrak{S}\left(\mathfrak{D}^{(\lambda)},{ }^{*}\right)$.

Furthermore,

$$
\begin{aligned}
\mu^{*} & =\left\{\lambda(2 \lambda-1)^{-1}\right\}^{*}=\lambda^{*}\left(2 \lambda^{*}-1\right)^{-1}=(1-\lambda)(1-2 \lambda)^{-1} \\
& =(\lambda-1)(2 \lambda-1)^{-1}=\{(2 \lambda-1)-\lambda\}(2 \lambda-1)^{-1}=1-\mu,
\end{aligned}
$$

so in $\mathfrak{D}=\mathfrak{A}_{\Omega}^{(\mu)}$ we have $\left(x \cdot{ }_{\mu} y\right)^{*}=\mu^{*} x^{*} y^{*}+\left(1-\mu^{*}\right) y^{*} x^{*}=(1-\mu) x^{*} y^{*}+\mu y^{*} x^{*}$ $=y^{*} \cdot{ }_{\mu} x^{*}$; in other words, ${ }^{*}$ is an involution of second kind on $\mathfrak{D}$. This shows $\mathfrak{A}$ is as in case (ii).

Conversely, suppose $\mathfrak{A}$ is $\mathfrak{D}^{(\lambda)}$ or $\mathfrak{E}\left(\mathfrak{D}^{(\lambda)},{ }^{*}\right)$. Clearly $\mathfrak{A}$ is a (split) quasi-associative algebra in the first case; in the second, we have $\mathscr{D}=\lambda \mathfrak{S}\left(\mathfrak{D},{ }^{*}\right)+(1-\lambda) \mathfrak{H}\left(\mathfrak{D},{ }^{*}\right)$ (since $\left.\lambda+\lambda^{*}=1\right) \cong \mathfrak{S}(\mathfrak{D}, *)_{\Omega}$ for $\Omega=\Phi(\lambda)$, so $\mathfrak{D}^{(\lambda)}=\mathfrak{S}(\mathfrak{D}, *)_{\Omega}^{(\lambda)}=\mathfrak{S}_{\mathcal{E}}\left(\mathfrak{D}^{(\lambda)}, *\right)_{\Omega}=\mathfrak{U}_{\Omega}$ and $\mathfrak{A}$ is quasi-associative.

From this we obtain

THEOREM 3. A noncommutative Jordan algebra over a field $\Phi$ is a quasi-associative division algebra if and only if it is isomorphic to one of

(i) $\Delta^{(\lambda)}$,

(ii) $\mathfrak{S}\left(\Delta^{(\lambda)}, *\right)$ for * an involution of second kind on $\Delta$, with $\lambda+\lambda^{*}=1$, where $\Delta$ is an associative division algebra over $\Phi$ and $\lambda \neq \frac{1}{2}$ an element in its center with $\lambda(1-\lambda) \in \Phi$. 
Proof. It is easy to see such algebras (i), (ii) are noncommutative Jordan division algebras (since $\mathfrak{A}^{+}$is a quadratic Jordan division algebra of the form $\Delta^{+}$or $\mathfrak{S}(\Delta, *))$. Conversely, if $\mathfrak{A}=\mathfrak{D}^{(\lambda)}$ is a noncommutative Jordan division algebra then $\mathfrak{U}^{+}=\mathfrak{D}^{+}$is a quadratic Jordan division algebra; since invertibility in $\mathfrak{D}$ and $\mathfrak{D}^{+}$ coincide, $\mathfrak{D}=\Delta$ is an associative division algebra. Similarly, if $\mathfrak{A}=\mathfrak{S}\left(\mathfrak{D}^{(\lambda)},{ }^{*}\right)$ is a division algebra so is $\mathfrak{A}^{+}=\mathfrak{S}\left(\mathfrak{D},{ }^{*}\right)$. But by the Herstein-Kleinfeld-Osborn Theorem [3, p. 166] this implies $\mathfrak{D}=\Delta \oplus \Delta^{\circ}$ (with exchange involution) or $\mathfrak{D}=\Delta$ (with involution) for $\Delta$ an associative division algebra, or else $\mathfrak{D}=\Phi_{2}$ is a split quaternion algebra with standard involution. In the latter case the involution is of first kind, so we can only have $\mathfrak{D}=\Delta \oplus \Delta^{\circ}$ or $\mathfrak{D}=\Delta$. The first of these leads back to (i); here $\lambda=\mu \oplus(1-\mu)^{\circ}$ and $\mathfrak{S}\left(\left(\Delta \oplus \Delta^{\circ}\right)^{(\lambda)},{ }^{*}\right) \cong \Delta^{(\mu)}$. The second leads to (ii): $\mathfrak{S}\left(\Delta^{(\lambda)},{ }^{*}\right)$.

3. Forms of algebras of degree 2. For the degree 2 algebras $\mathfrak{A}(Q, S, \times)$ of type (III) the case of characteristic 2 must be treated separately. We begin with the characteristic $\neq 2$ case. A form of an algebra of generic degree 2 is itself of degree 2 , $a^{2}-T(a) a+N(a) 1=0$ for $T, N$ the trace and norm. Here $\mathfrak{A}^{+}=\Phi 1+\mathfrak{B}$ is the Jordan algebra of a quadratic form $Q$ on $\mathfrak{B}, \mathfrak{B}$ being the space of elements of trace zero, with $T(a)=2 \alpha, N(a)=\alpha^{2}-Q(v)$ for $a=\alpha 1+v \quad(v \in \mathfrak{B})$. The noncommutative product is determined by the rule that 1 acts as identity and $v w=v \cdot w+v \times w$ for $v, w \in \mathfrak{B}$, where $v \cdot w=\frac{1}{2}(v w+w v)=\frac{1}{2}\{Q(v+w)-Q(v)-Q(w)\} 1=\sigma(v, w) 1$ is a symmetric bilinear form with $\sigma(v, v)=Q(v)$ and $v \times w=\frac{1}{2}(v w-w v)$ an alternate bilinear product on $\mathfrak{B}$. Flexibility implies $v \cdot[v, w]=[v, v \cdot w]=0$ for $v, w \in \mathfrak{B}$, or $\sigma(v, v \times w)$ $=0$. By linearization, $\sigma(u \times w, v)=\sigma(v, u \times w)=-\sigma(u, v \times w)=\sigma(u, w \times v)$ and $\sigma$ is associative. (Conversely, if $\sigma$ is associative then $\sigma(v, v \times w)=\sigma(v \times v, w)=0$.)

Theorem 4. Let $\Phi$ be a field of characteristic $\neq 2$. The noncommutative Jordan algebras of generic degree 2 are precisely the algebras $\mathfrak{A}(\sigma, \times)=\Phi 1+\mathfrak{B}$ where multiplication is given by

$$
v w=\sigma(v, w) 1+v \times w \quad(v, w \in \mathfrak{B})
$$

where $v \times w$ is an alternate product on $\mathfrak{B}, v \times v=0$, and $\sigma$ is a symmetric associative bilinear form on $\mathfrak{B}, \sigma(u \times v, w)=\sigma(u, v \times w) . \mathfrak{A}(\sigma, \times)$ is a division algebra if and only if the quadratic form $Q(v)=\sigma(v, v)$ does not represent 1 or 0 .

Indeed, $\mathfrak{A}$ is a division algebra if and only if $N(a) \neq 0$ for $a \neq 0$, or $Q(v) \neq \alpha^{2}$ does not represent a square unless $v=0, \alpha=0$, and this is equivalent to saying $Q$ does not represent 1 or 0 .

Now we turn to the characteristic 2 case. Suppose $\mathfrak{A}$ is a form of an algebra $\mathfrak{A}(Q, S, \times)$ of type (III). Then $\mathfrak{A}$ is of degree $2, a^{2}-T(a)+Q(a) 1=0$, where $T(a)$ $=Q(a, 1)$ is not identically zero since $Q(a, b)$ is nondegenerate. Therefore we can choose $t \in \mathfrak{A}$ with $T(t)=1$. (Note $T(1)=2=0$ in the characteristic 2 case, and also $Q(a, a)=2 Q(a)=0$.) Thus we have $1, t$ with $Q(1,1)=Q(t, t)=0, Q(1, t)=1$. Splitting off this hyperbolic subspace, we can write $\mathfrak{A}=\Phi \mathbf{1} \oplus \Phi t \oplus \mathfrak{B}$ for $\mathfrak{B}=\{v \mid Q(1, v)=Q(t, v)=0\}$. Clearly $t^{2}=T(t) t+Q(t) 1=t+\tau 1$ for $\tau=Q(t)$. The 
other multiplications are not so easy to establish. (Things would be much simpler if we could take $t$ to be an idempotent, as in $\mathfrak{U}(Q, S, \times)=\Phi e_{1} \oplus \Phi e_{2} \oplus \mathfrak{B}$, but we are working with the general nonsplit case.)

First, we claim $t \mathfrak{B} \subset \mathfrak{B}$. By flexibility, $t \circ t v=t(t \circ v)$. Now $t \circ v=T(t) v+T(v) t$ $+Q(t, v) 1=v$, so $t(t \circ v)=t v$, and $t \circ t v=T(t) t v+T(t v) t+Q(t, t v) 1=t v+T(t v) t$ $+Q(t, t v) 1$. Thus $Q(1, t v) t+Q(t, t v) 1=0$, and by directness $Q(1, t v)=Q(t, t v)=0$, which means $t v \in \mathfrak{B}$. Thus $L_{t}$ induces a linear transformation $S$ on $\mathfrak{B}$, and from $t \circ v=v$ we have $L_{t}+R_{t}=I$ :

$$
t v=S(v), \quad v t=(I-S)(v) \quad(v \in \mathfrak{B}) .
$$

For $v, w \in \mathfrak{B}$ write

$$
v w=f(v, w) 1+g(v, w) t+v \times w
$$

where $f, g$ are bilinear forms and $v \times w$ a bilinear product on $\mathfrak{B}$. From $v^{2}=T(v) v$ $+Q(v) 1=Q(v) 1$ we see

$$
f(v, v)=Q(v), \quad g(v, v)=0, \quad v \times v=0 \quad(v \in \mathfrak{B}) .
$$

As before, flexibility gives $Q(v, w) v=v\{T(v) w+T(w) v+Q(v, w) 1\}=v(v \circ w)=v \circ v w$ $=T(v) v w+T(v w) v+Q(v, v w) 1=T(v w) v+Q(v, v w) 1$. Since by $(4), T(v w)=g(v, w)$, $Q(v, v w)=Q(v, v \times w)$, we obtain

$$
g(v, w)=Q(v, w), \quad Q(v, v \times w)=0 \quad(v, w \in \mathfrak{B}) .
$$

Similarly,

$$
\begin{aligned}
Q(v w, t) 1+T(v w) t+v w+Q(t w, v) 1 & =t \circ v w+v \circ t w=t(v \circ w)+v(t \circ w) \\
& =Q(v, w) t+v w
\end{aligned}
$$

by flexibility; identifying coefficients of 1 we obtain $Q(v w, t)=Q(t w, v)$. But $Q(v w, t)=f(v, w)$ by (4), and $t w=S(w)$, so $f(v, w)=Q(S w, v)$. This establishes the first part of

THEOREM 5. Let $\Phi$ be a field of characteristic $2 . \mathfrak{A}$ is a noncommutative Jordan algebra of generic degree 2 with nonzero trace if and only if $\mathfrak{A}$ is an algebra $\mathfrak{A}(Q, S, \times, \tau)=\Phi 1 \oplus \Phi t \oplus \mathfrak{B}$ with multiplication

$$
\begin{aligned}
t^{2} & =t+\tau 1 \quad(\tau=Q(t), 1=T(t)), \\
t v & =S(v), \quad v t=(I-S)(v), \\
v w & =Q(v, S w) 1+Q(v, w) t+v \times w \quad(v, w \in \mathfrak{B})
\end{aligned}
$$

where $Q$ is a quadratic form, $S$ a linear transformation, and $v \times w$ an alternate bilinear product on $\mathfrak{B}$ satisfying $Q(S v, v)=Q(v)$ and $Q(v, v \times w)=0$ for all $v, w \in \mathfrak{B}$.

$\mathfrak{U}(Q, S, \times, \tau)$ is a division algebra if and only if $Q$ does not represent 1,0 , or any $\gamma^{2}+\gamma+\tau$ for $\gamma \in \Phi$. 
The converse is straightforward: $\mathfrak{A}$ has generic degree 2 , with $T(\alpha 1+\beta t+v)=\beta$ and $N(\alpha 1+\beta t+v)=\alpha^{2}+\alpha \beta+\beta^{2} \tau+Q(v)$, and is flexible (it suffices if $a \circ a b=a(a \circ b)$ for $a, b \in \Phi t+\mathfrak{B}$, which reduces to $N(a, b)=T(a) T(b)+T(a b)$ and $N(a b, a)$ $=T(b) N(a)$ for such $a$ and $b$, and these can be verified using $T(v w)=Q(v, w)$, $N(v w, v)=0, N(w v, t)=Q(t v, w), Q(t v, v)=Q(v))$.

The condition $N(a) \neq 0$ for $a \neq 0$ that $\mathfrak{A}$ be a division algebra becomes $Q(v) \neq \alpha^{2}$ $+\alpha \beta+\beta^{2} \tau$ unless $\alpha=\beta=v=0$. If $\beta=0$ this means $Q(v) \neq \alpha^{2}$, or $Q$ does not represent 1 or 0 ; if $\beta \neq 0$ it means $(\alpha / \beta)^{2}+(\alpha / \beta)+\tau \notin Q(\mathfrak{B})$. Thus the necessary and sufficient condition that $\mathfrak{A}$ be a division algebra is that $Q$ represent neither 1,0 , nor any $\gamma^{2}+\gamma+\tau$ for $\gamma \in \Phi$.

4. Forms of nodal algebras. Once again the characteristic 2 case must be handled separately. We consider first type (IIa).

TheOREM 6. Let $\Phi$ be a field of characteristic $p \neq 0,2$. A noncommutative Jordan algebra $\mathfrak{A}$ is a division algebra form of a nodal algebra $\mathfrak{A}\left(F_{n}, C\right)$ of type (IIa) if and only if $\mathfrak{A}$ is an algebra $\mathfrak{A}(\Sigma, D)$ which is obtained from a purely inseparable extension field $\Sigma=\Phi\left(x_{1}, \ldots, x_{n}\right)$ of $\Phi$ of exponent $1, x_{i}^{p}=\alpha_{i} \in \Phi$, by means of the product

$$
x y=x \cdot y+x \times y, \quad x \times y=\sum_{i, j} d_{i j} \cdot \partial_{i} x \cdot \partial_{j} y,
$$

where $x \cdot y$ is the commutative associative product in $\Sigma, \partial_{i}=\partial / \partial x_{i}$ denotes partial derivation with respect to $x_{i}$, and $d_{i j} \in \Sigma$ satisfy $d_{i j}+d_{j_{i}}=0$.

Proof. We follow a standard argument [12, pp. 143-147]. Since $\mathfrak{A}_{\Omega}=\mathfrak{A}\left(F_{n}, C\right)$ $=\Omega 1+\mathfrak{N}$ is nodal for some $\Omega$ if $\mathfrak{A}$ is a form of an algebra of type (IIa), $\mathfrak{A}_{\Omega}^{+}$is associative and therefore $\mathfrak{A}^{+}$is too. Thus $\mathfrak{A}^{+}$is a commutative associative division algebra, i.e. an ordinary field $\Sigma$. Any $x \in \mathfrak{U}$ has the form $x=\omega 1+z$ for $\omega \in \Omega$, $z \in \mathfrak{N}$ in $\mathfrak{A}_{\Omega}$, hence $x^{p}=\omega^{p} 1+z^{p}=\omega^{p} 1 \in \mathfrak{A} \cap \Omega 1=\Phi 1$, and $\omega^{p}=\alpha \in \Phi$. This shows $\Sigma$ is purely inseparable of exponent 1 , so we can find generators $x_{1}, \ldots, x_{n}$ with

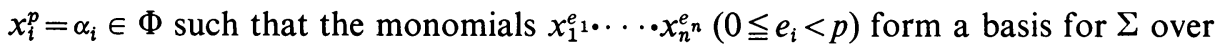
$\Phi$. Then, as in [12, p. 144], multiplication in $\mathfrak{A}$ is given by $x y=x \cdot y+x \times y$ for $x \cdot y$ $=\frac{1}{2}(x y+y x)$ the ordinary product in $\mathfrak{A}^{+}=\Sigma$ and $x \times y=\frac{1}{2}[x, y]=D_{x}(y)$ given by

$$
x \times y=\sum d_{i j} \cdot \partial_{i} x \cdot \partial_{j} y \quad\left(d_{i j}+d_{j i}=0\right)
$$

for certain coefficients $d_{i j} \in \Sigma$. Conversely, every such algebra $\mathfrak{A}(\Sigma, D)$ is a noncommutative Jordan algebra which is a division algebra since $\mathfrak{A}(\Sigma, D)^{+}=\Sigma$, and is a form of $\mathfrak{U}\left(F_{n}, C\right)$ since $\Sigma_{\Sigma}=\Sigma \otimes \Sigma=\Sigma\left[z_{1}, \ldots, z_{n}\right]=F_{n}$ where $z_{i}=1 \otimes x_{i}$ $-\alpha_{i}^{1 / p} \otimes 1$ (note that this actually implies $c_{i j}=\frac{1}{2}\left[z_{i}, z_{j}\right]=\frac{1}{2}\left[x_{i}, x_{j}\right]=d_{i j}$ ).

REMARK. It is more difficult to give conditions under which such an algebra will be central. It is not hard to show the center of $\mathfrak{A}(\Sigma, D)$ is the set of $z$ such that $z \times \mathfrak{A}=0$, i.e. $D_{z}=0$. The above $\mathfrak{A}$ will be central if and only if $\mathfrak{A}_{\Omega}$ is simple. However, no necessary and sufficient conditions on the coefficients $c_{i j}$ in $\mathfrak{A}\left(F_{n}, C\right)$ $=\Omega\left[z_{1}, \ldots, z_{n}\right]=\Omega 1+\mathfrak{N}\left(z_{i}^{p}=0\right)$ are known in order that it be simple. Writing 
$c_{i j}=\alpha_{i j} 1+z_{i j}\left(\alpha_{i j} \in \Omega, z_{i j} \in \mathfrak{\Re}\right)$ it is clearly necessary that at least one $\alpha_{i j} \neq 0$ so that $\mathfrak{N}$ is not a subalgebra (consequently a nil ideal).

It is also easy to see it is sufficient if the determinant det $\left(\alpha_{i j}\right)$ of the coefficients is nonzero (which is possible only if $n$ is even, since the matrix $\left(\alpha_{i j}\right)$ is skew). Indeed, since $a \rightarrow a^{p}$ is a homomorphism on $\mathfrak{A}\left(F_{n}, C\right)^{+}$we have $\left\{\operatorname{det}\left(\alpha_{i j}\right)\right\}^{p}=\operatorname{det}\left(\alpha_{i j}^{p}\right)$ $=\operatorname{det}\left(c_{i j}^{p}\right)=\left\{\operatorname{det}\left(c_{i j}\right)\right\}^{p}$ so that if the matrix $\left(\alpha_{i j}\right)$ has nonzero determinant, so does the matrix $\left(c_{i j}\right)$. To prove $\mathfrak{A}\left(F_{n}, C\right)$ is simple in this case, we must show there is no proper $\mathfrak{A}\left(F_{n}, C\right)^{+}$-subspace $\mathfrak{B}$ with $\mathfrak{A}\left(F_{n}, C\right) \times \mathfrak{B} \subset \mathfrak{B}$ (equivalently, $D_{z_{k}}(\mathfrak{B}) \subset \mathfrak{B}$ for $k=1, \ldots, n)$. If $\left(d_{i j}\right)$ is the inverse of $\left(c_{i j}\right)$ then for all $x \in \mathfrak{B}$ the element $\sum_{k} d_{i k} \cdot D_{z_{k}}(x)=\sum_{k, j} d_{i k} \cdot c_{k j} \cdot \partial_{j}(x)=\partial_{i}(x)$ would belong to the invariant subspace $\mathfrak{B}$ for $i=1, \ldots, n$. But then $\mathfrak{B}$ would be invariant under the partial derivatives $\partial_{i}$, and this implies $\mathfrak{B}=0$ or $\mathfrak{B}=\mathfrak{A}\left(F_{n}, C\right)$ because if $x \neq 0$ in $\mathfrak{B}$ has nonzero coefficient

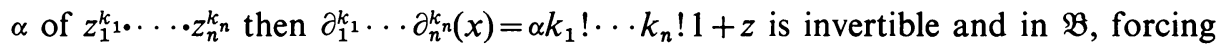
$\mathfrak{B}=\mathfrak{A}\left(F_{n}, C\right)$. This sufficiency condition is due to L. Kokoris [4, p. 491]. From the work of J. Goldman [2] it is known the condition is not necessary.

We now turn to case (IIb).

THEOREM 7. Let $\Phi$ be a field of characteristic 2. A noncommutative Jordan algebra $\mathfrak{A}$ is a form of an algebra $\mathfrak{A}(\tau, \times)$ of type (IIb) if and only if $\mathfrak{A}$ is an algebra $\mathfrak{A}(\sigma, \times)$ $=\Phi 1 \oplus \mathfrak{B}$ with product

$$
v w=\sigma(v, w) 1+v \times w \quad(v, w \in \mathfrak{B})
$$

where $\sigma$ is a (not necessarily alternate) symmetric bilinear form and $v \times w$ an alternate bilinear product on $\mathfrak{B}$.

$\mathfrak{2}(\sigma, \times)$ is a form of a nodal algebra if and only if $\sigma(v \times w, v \times w) \not \equiv \sigma(v, w)^{2}$ $+\sigma(v, v) \sigma(w, w) . \mathfrak{H}(\sigma, \times)$ is a division algebra if and only if $\sigma(v, v)$ does not represent 1 or 0.

Proof. Suppose first $\mathfrak{A}$ is a form of an algebra of type (IIb), $\mathfrak{A}_{\Omega}=\mathfrak{A}(\tau, \times)=\Omega 1$ $+\mathfrak{N}$. Since we have $a^{2}=\omega^{2} 1 \in \Omega 1$ for any $a=\omega 1+z$ in $\mathfrak{A}_{\Omega}$, we have $a^{2}=Q(a) 1 \in \Phi 1$ for any $a \in \mathfrak{A}$. Here $Q$ is a quadratic form on $\mathfrak{A}$ with values in $\Phi$ such that $Q(a, b) 1$ $=\{Q(a+b)-Q(a)-Q(b)\} 1=(a+b)^{2}-a^{2}-b^{2}=a b+b a=2 a b=0$ by commutativity in characteristic 2 .

Write $\mathfrak{A}=\Phi 1 \oplus \mathfrak{B}$ where $\mathfrak{B}$ is any vector-space complement of $\Phi 1$, and let $E, F$ be the corresponding projections on $\Phi 1$ and $\mathfrak{B}$. Here

$$
v w=\sigma(v, w) 1+v \times w \quad(v, w \in \mathfrak{B})
$$

for $\sigma(v, w) 1=E(v w)$ and $v \times w=F(v w) \in \mathfrak{B}$. Clearly $\sigma(v, w)$ is a $\Phi$-bilinear form on $\mathfrak{B}$, which is symmetric since $\mathfrak{A}$ is commutative, and $v \times w$ is a bilinear product. From $v^{2}=\sigma(v, v) 1+v \times v=Q(v) 1$ we conclude

$$
\sigma(v, v)=Q(v), \quad v \times v=0,
$$

so $v \times w$ is alternate. 
Conversely, we claim any such $\mathfrak{A}=\mathfrak{A}(\sigma, \times)$ is a form of an $\mathfrak{U}(\tau, \times)$. Note that $\mathfrak{A}$ is commutative and $a^{2}=Q(a) 1$ for any $a \in \mathfrak{A}$, and these remain valid in any extension $\mathfrak{A}_{\Omega}$. If we take $\Omega$ to be the algebraic closure of $\Phi$ then in $\mathfrak{A}_{\Omega}$ we may write any element as $a=\omega 1+z$ for $\omega=Q(a)^{1 / 2}$, in which case $z^{2}=(a-\omega 1)^{2}=a^{2}-\omega^{2} 1$ $=Q(a) 1-Q(a) 1=0$. Thus $\mathfrak{A}_{\Omega}=\Omega 1+\mathfrak{N}$ where $\mathfrak{N}=\left\{z \mid z^{2}=0\right\}=\{z \mid Q(z)=0\}$ is a subspace since $Q(z+w)=Q(z)+Q(w)$. From this we see as before that $\mathfrak{A}_{\Omega}=\mathfrak{A}(\tau, \times)$ where $z w=\tau(z, w) 1+z \times w(z, w \in \mathfrak{R})$ with $\tau$ and $z \times w$ both alternate (because $z^{2}=0$ for $\left.z \in \mathfrak{R}\right)$.

The algebras $\mathfrak{A}(\tau, \times)=\Omega 1+\mathfrak{N}$ are always almost-nil; we claim such an algebra is nodal if and only if the form $Q$ does not permit composition, $Q(a b) \not \equiv Q(a) Q(b)$ $\left(a^{2}=Q(a) 1\right)$. Indeed, since $\mathfrak{N}=\{z \mid Q(z)=0\}$ the relation $Q(a b)=Q(a) Q(b)$ would imply the subspace $\mathfrak{N}$ was closed under products, hence an ideal, and $\mathfrak{A}(\tau, \times)$ would not be nodal. Conversely, if $\mathfrak{A}(\tau, \times)$ is not nodal, so $\mathfrak{N}$ is an ideal, then for $a=\alpha 1+z, b=\beta 1+w$ we have $a b=\alpha \beta 1+v(v=\alpha w+\beta z+z w \in \mathfrak{R})$ so $Q(a b)=(\alpha \beta)^{2}$ $=\alpha^{2} \beta^{2}=Q(a) Q(b)$.

Thus $\mathfrak{A}=\mathfrak{A}(\sigma, \times)$ will be a form of a nodal algebra $\mathfrak{A}_{\Omega}=\mathfrak{A}(\tau, \times)$ unless $Q(a b)$ $\equiv Q(a) Q(b)$ on $\mathfrak{A}$ (hence $\mathfrak{A}_{\Omega}$ ). We want to rephrase this in terms of $\sigma$. For $a=\alpha 1+v$, $b=\beta 1+w, a b=\{\alpha \beta+\sigma(v, w)\} 1+\{\alpha w+\beta v+v \times w\}$ we have

$$
\begin{aligned}
Q(a b) & =\alpha^{2} \beta^{2}+\sigma(v, w)^{2}+\alpha^{2} Q(w)+\beta^{2} Q(v)+Q(v \times w) \\
& =\left\{\alpha^{2}+Q(v)\right\}\left\{\beta^{2}+Q(w)\right\}+\sigma(v, w)^{2}+Q(v \times w)-Q(v) Q(w) \\
& =Q(a) Q(b)+\sigma(v, w)^{2}+\sigma(v \times w, v \times w)+\sigma(v, v) \sigma(w, w),
\end{aligned}
$$

so $\mathfrak{A}(\tau, \times)$ will be nodal unless

$$
\sigma(v \times w, v \times w) \equiv \sigma(v, v) \sigma(w, w)+\sigma(v, w)^{2} .
$$

Since $a^{2}=Q(a) 1$ for $Q(\alpha 1+v)=\alpha^{2}+\sigma(v, v)$, it is clear that $\mathfrak{A}(\sigma, \times)$ is a division algebra if and only if $Q(a) \neq 0$ for $a \neq 0$, which means $Q(v) \neq \alpha^{2}$ unless $\alpha=v=0$, or equivalently $Q(v)=\sigma(v, v)$ does not represent 1 or 0 .

REMARK. Nodality of $\mathfrak{A}(\tau, \times)$ is also equivalent to $\tau \neq 0$, but since $\tau$ is not the extension of $\sigma(\mathfrak{N}$ need not be the extension of $\mathfrak{B})$ this condition is not so easily translated into a condition on $\sigma$.

Also notice the formal similarity between these algebras $\mathfrak{A}(\sigma, \times)$ and the $\mathfrak{A}(\sigma, \times)$ of Theorem 4; in some ways these characteristic 2 nodal algebras are traceless versions of degree 2 algebras $\left(a^{2}-T(a) a+Q(a) 1=0\right.$ becomes $a^{2}=Q(a) 1$ in characteristic 2 when $T=0$ ).

\section{REFERENCES}

1. A. A. Albert, A construction of exceptional Jordan division algebras, Ann. of Math. (2) 67 (1958), 1-28. MR 19, 1036.

2. J. Goldman, Nodal algebras of dimension $p^{3}$, Proc. Amer. Math. Soc. 24 (1970), 156-160.

3. N. Jacobson, Structure and representations of Jordan algebras, Amer. Math. Soc. Colloq. Publ., vol. 39, Amer. Math. Soc., Providence, R. I., 1968. MR 40 \#4330.

4. L. Kokoris, Nodal non-commutative Jordan rings, Canad. J. Math. 12 (1960), 488-492. MR 22 \#6834. 
5. K. McCrimmon, Noncornmutative Jordan rings, Trans. Amer. Math. Soc. 158 (1971), $1-33$.

6. - A note on quasi-associative algebras, Proc. Amer. Math. Soc. 17 (1966), 1455-1459. MR 39 \#270.

7. K. McCrimmon and R. D. Schafer, On a class of noncommutative Jordan algebras, Proc. Nat. Acad. Sci. U.S.A. 56 (1966), 1-4. MR 34 \#5888.

8. K. McCrimmon, Quadratic Jordan algebras and cubing operations, Trans. Amer. Math. Soc. 153 (1971), 265-278.

9. - - Homotopes of noncommutative Jordan algebras, Math. Ann. 191 (1971), 263-270.

10. — The Freudenthal-Springer-Tits constructions of exceptional Jordan algebras, Trans. Amer. Math. Soc. 139 (1969), 495-510. MR 39 \#276.

11. - The Freudenthal-Springer-Tits constructions revisited, Trans. Amer. Math. Soc. 148 (1970), 293-314.

12. R. D. Schafer, An introduction to nonassociative algebras, Pure and Appl. Math., vol. 22, Academic Press, New York, 1966. MR 35 \#1643.

Department of Mathematics, University of Virginia, Charlottesville, Virginia 22903 